\title{
MedienPädagogik
}

Themenheft Nr. 27: Tagungsband: Spannungsfelder und blinde Flecken.

Medienpädagogik zwischen Emanzipationsanspruch und Diskursvermeidung. Herausgegeben von Sven Kommer, Thorsten Junge und Christiane Rust.

\section{Warum Computerspielen trotzdem gut ist Neutralisierungsstrategien von Computerspielabhängigen und sozialwissen- schaftlichen Forschern}

Paula Bleckmann und Nadine Jukschat

\section{Zusammenfassung}

Im folgenden Text werden zunächst verschiedene wissenschaftliche Perspektiven auf das Phänomen der Computerspielabhängigkeit vorgestellt und die Position der Autorinnen, die Computerspielabhängigkeit in der Tradition sozialwissenschaftlicher Suchtforschung als virtuelle (Schein-)Lösung verschiedener biografischer Problemkonstellationen sowie als unwahrscheinlichen Prozess verstehen, hiervon abgegrenzt. Zweitens werden innerhalb des als «unwahrscheinliche Karriere» verstanden Prozesses verschiedene hier als Neutralisierungsstrategien bezeichnete Deutungsmuster in den Fokus genommen, die zu einem Nichtergreifen von Karriereabbruchmöglichkeiten führen. Es sind vielfältige Neutralisierungsstrategien aus dem Material rekonstruierbar, mit denen Spieler/innen plausibilisieren, «warum Computerspielen trotzdem gut ist», trotz der Konflikte also, die sich zunehmend mit Normen und Werten des sozialen Umfelds oder der Gesellschaft ergeben. Dabei ist zwischen offensiveren und defensiveren, wie auch zwischen jene Normen aushebelnden oder aber diese anerkennenden Strategien zu unterscheiden, wobei verschiedene Strategien auch zugleich von ein und derselben Person eingesetzt werden. Drittens wird die Frage aufgeworfen, inwieweit die Argumentationslinien der durch Kommerzialisierungsinteressen getriebenen Medienanbieter, wie auch der Medienpädagogik und ihrer Nachbardisziplinen Parallelen zu diesen Neutralisierungsstrategien aufweisen. Kann diese gewagte Parallelisierung die Medienpädagogik zu einer kritischen Selbstreflexion anregen, um sich in einem Diskurs um Medienrisiken reflektierter positionieren zu können, statt Hinweise zu Risiken der Bildschirmmediennutzung, die vorwiegend aus anderen Disziplinen stammen, entweder zu ignorieren oder aber reflexartig zu neutralisieren, indem begründet wird, «warum Computerspielen trotzdem gut» sei? 


\title{
Why gaming is good nonetheless - strategies of neutralization among video game addicts and researchers in the social sciences
}

\begin{abstract}
In a first step, different current approaches toward describing the phenomenon of video game addiction are briefly characterized, including the authors' own position: Understanding it, in the tradition of socio-scientific addiction research, as a virtual (mock) solution for different biographical problem constellations and an unlikely outcome in a process model. Secondly, we describe how a certain type of attribution pattern, which we call strategies of neutralization, contributes to the non-exploitation of chances to abandon the unlikely addiction career. A multitude of different strategies of neutralization can be reconstructed from interviews with (addicted) gamers which corroborate the attribution that "gaming is good nonetheless», i.e. in spite of the increasing conflicts with norms and values of their immediate social and larger societal environment. The strategies can be classified according to whether they are more aggressive or defensive, whether they tend to accept or lever out these norms and values. One interviewee is often found to use several strategies in parallel. Thirdly, we investigate to what extent parallels to these strategies of neutralizing can be found in the structure of arguments used by media providers driven by commercial interests, but also by media education research and practice and its neighbouring disciplines. Can this daring comparison assist a critical self-reflection within the media education scene and lead to a more reflected self-positioning in the face of concerns about screen media risks which mainly originate from other disciplines, instead of ignoring or rejecting them in a knee-jerk impulse to argue «why gaming is good nonetheless»?
\end{abstract}

\section{Einleitung}

Der erste Teil des folgenden Beitrags wird innerhalb eines qualitativ-empirisch fundierten Modells suchtartiger Computerspielpraxis verschiedene Neutralisierungsstrategien charakterisieren und voneinander abgrenzen, welche von Computerspielenden am kritischen Punkt eines möglichen Karriereabbruchs bewusst oder unbewusst eingesetzt werden. Die Neutralisierungsstrategien begleiten und ermöglichen dabei die Fortsetzung und Ausweitung der Computerspielpraxis, indem sie plausibilisieren helfen, «warum Computerspielen trotzdem gut ist». Dieser erste Teil könnte gut für sich allein stehen, oder nur durch einen kurzen Hinweis ergänzt werden, dass ein Vergleich dieser individuellen Neutralisierungsstrategien mit den Argumentationslinien «im Grossens lohnend sein könnte, die sich in der öffentlichen Debatte in den Medien, in den Darstellungen einzelner grosser Medienund insbesondere Computerspielanbieter oder der Verbände kleinerer Anbieter 
finden, in denen heute ebenfalls mit Nachdruck und Raffinesse begründet wird, "warum Computerspielen trotzdem gut ist», also trotz der sich mehrenden Hinweise auf kontraproduktive und problematische Nutzungsformen und ihre Auswirkungen. Anstelle dieses einfachen und innerhalb der Medienpädagogik vermutlich auf breite Akzeptanz stossenden Wegs der Kritik am teilweise stark vereinseitigenden und manipulativen «Public Perception Management» grosser Konzerne wird das Wagnis eingegangen, in einem zweiten Teil dieses Beitrags exemplarisch Parallelen zu den individuellen Neutralisierungsstrategien zu benennen und zu diskutieren, die nicht nur aus dem Bereich der Argumentationslinien der Konzerne, sondern auch in der wissenschaftlichen Debatte innerhalb der Medienpädagogik und ihren Nachbardisziplinen zu finden sind. Bei einem solchen Wagnis ist Vorsicht geboten, so dass bereits an dieser Stelle erste Hinweise auf die unten noch genauer zu erläuternden Limitationen dieser Vorgehensweise erlaubt seien: Während die Neutralisierungen im ersten Teil innerhalb eines fundierten empirischen oder theoretischen Gesamtrahmens dargestellt werden, setzt der zweite Teil lediglich einzelne provokante Impulse als Einladung und Anregung zum Weiterdenken. Schliesslich noch eine wichtige Klärung vorab: Neutralisierungsstrategien werden hier anhand ihrer Funktion zur Verhinderung eines Karriereabbruchs als solche einsortiert, womit gerade nicht geklärt ist, ob sie sich im weiteren biographischen Verlauf normativ als hilfreich oder problemverschärfend erweisen werden.

\section{Deutungsmodelle suchtartigen Computerspielverhaltens.}

Im Jahr 2013 wurde suchtartiges Computerspielen unter der Bezeichnung IGD (Internet Gaming Disorder) als Forschungsdiagnose im DSM-5 aufgenommen (American Psychiatric Association 2013). Das Forschungsfeld wird derzeit durch eine quantitative, pathogenetisch ${ }^{1}$ orientierte medizinisch-psychologische Suchtforschung beherrscht, die im Einzelfall so weit geht, dass man hofft, die Krankheit durch Psychopharmaka neuropsychologisch therapieren zu lernen: «By discovering the brain molecules that control the development of [...] addiction, we hope to identify new treatment approaches» (O'Brien 2014). Unter weitgehender Ausblendung bzw. Vernachlässigung der Analyse von Mediensozialisationsforschung, In-Game-Verhaltensweisen und der ausgedehnten Literatur dazu droht sich eine Idee von Computerspielsucht als Krankheit, gar als Hirnfunktionsstörung, die von Experten therapiert werden müsse, als dominantes Deutungsmuster für dieses neue Phänomen zu etablieren (vgl. Jukschat 2013).

In der Forschungstradition der Games Studies dagegen werden In-Game-Verhaltensweisen und Spielmotivation aus der Sicht der Spieler differenziert beschrieben, auch mit ethnographischen Methoden als neue Kulturform, ja gar als subversive

1 Die Pathogenese beschreibt die Entstehung und Entwicklung einer Krankheit. 
Subkulturen charakterisiert wie z. B. im World of Warcraft Reader (Corneliussen/ Rettberg 2008). Die Biographie der Computerspielenden ausserhalb ihrer InGame-Aktivitäten wird dabei in der Regel kaum einbezogen. Die Existenz des Phänomens Computerspielsucht bzw. Internetsucht wurde innerhalb der Games Studies und den ihr nahe stehenden Teildisziplinen der Medienpädagogik eher prinzipiell in Frage gestellt und allenfalls aus einer konstruktivistischen Perspektive betrachtet (Cover 2006; Dellwing 2013; Walter/Schetsche 2003; Wimmer 2013). Inzwischen erfolgt jedoch parallel dazu auch eine Auseinandersetzung mit den Phänomenen ausufernder Computerspielpraxis in (Medien-)Pädagogik und Sozialwissenschaften, bei der die Frage, ob es sich um eine Sucht handle, nicht im Zentrum steht, sondern die Charakterisierung des Erlebens der Computerspielenden und ihres sozialen Umfelds (Chappell et al. 2006; Domahidi/Quandt 2014; Hussain/Griffiths 2009; Kammerl et al. 2012; Schmidt et al. 2011). Dabei werden problematische Aspekte der Spielpraxis eher als Symptom denn als zugrundeliegendes Problem verstanden, z. B. als Reaktion auf kritische Lebensereignisse, als vorübergehende exzessive Nutzungsphase in der Adoleszenz oder als Symptom einer problematischen Familiensituation.

Eine zwischen Pathologisierung und Normalisierung vermittelnde Position nimmt die aufgrund biographischer Interviews innerhalb qualitativer Längsschnittstudien generierte Grounded Theory von Bleckmann und Jukschat (2015) ein: Das «integrierte (Dys-)Funktionalitätsmodell der suchtartigen Computerspielnutzung» beschreibt suchtartiges Computerspielverhalten als virtuelle (Schein-)Befriedigung der (Sehn-)Sucht nach Zugehörigkeit, nach Anerkennung für Leistungen und nach Autonomie, wobei es in der Regel durch das Computerspielverhalten gerade nicht zu einer Lösung, sondern einer Verschärfung der biographischen Problemlage kommt, entsprechend des Konzepts einer versuchten virtuellen Selbstmedikation in der Tradition der sozialwissenschaftlichen Suchtforschung (Dollinger/SchmidtSemisch 2007).

\section{Konfliktentstehung, Vertuschungs- und Neutralisierungsstrategien}

Im Folgenden sollen verschiedene Neutralisierungsstrategien im Fokus stehen, die innerhalb einer Computerspielabhängigkeitskarriere in dem Moment bedeutsam werden, in dem die entwickelte Spielpraxis in ihrer Intensität mit Ansprüchen der Gesellschaft und konstitutiven Erwartungen an Teilhabe des sozialen Umfeldes in Konflikt gerät und dies von anderen oder den Spielenden selbst bemerkt wird. Sie sind Teil eines auf derselben Datengrundlage wie das «integrierte (Dys-)Funktionalitätsmodell» erstellten komplexen Prozess- oder Karrieremodells, das sich an dem sozialwissenschaftlichen Konzept der Karriere (vgl. Becker 1963; Dollinger 2002; Gerhardt 1991; Goffman 1977) orientiert und die Entstehung und Verstetigung 
einer Computerspielabhängigkeit als unwahrscheinlichen Prozess begreift (Jukschat 2016). ${ }^{2}$ Hat der Spieler eine Spielpraxis entwickelt, die in ihrer Intensität mit Ansprüchen des sozialen Umfelds bzw. der Gesellschaft ernsthaft in Konflikt gerät, so stellt sich die Frage, warum an dieser Stelle nicht interne und externe soziale Kontrollinstanzen greifen und die Computerspielabhängigkeitskarriere nicht an diesem Punkt abbricht. Beispiele im Material für solche Konflikte sind Schul- oder Beziehungsschwierigkeiten, Gefährdung oder Verlust des Arbeitsplatzes, Kreislauf- und Schlafprobleme, starke Gewichtszunahme oder-abnahme und anderes mehr. Im Interviewmaterial werden eine Reihe kommunikativer und lebenspraktischer Techniken sichtbar, die das Konfliktpotenzial neutralisieren bzw. aushebeln, das sich durch die Abweichung von bestimmten geteilten Werten und Normen sowie dem Bruch mit an sie gerichteten konstitutiven Erwartungen ergibt. Diese Neutralisierungsstrategien wurden vorwiegend anhand von Interviews mit aktuell an diesem Punkt im Karriereverlauf befindlichen Personen ausgearbeitet und später durch Hinzunahme von Interviews mit ehemals suchtartig Spielenden erweitert. Isolations- bzw. Vertuschungsstrategien. Diese Strategien liegen auf der Handlungsebene und zielen darauf ab, ein Konfliktpotenzial gar nicht erst zum Tragen kommen zu lassen, indem eine Konfrontation mit Kontrollinstanzen vermieden wird. Bei allein lebenden, nicht berufstätigen Spieler/innen lassen sich vorwiegend solche Isolationsstrategien beschreiben, bei welchen realweltliche Sozialkontakte auf ein Mindestmass eingeschränkt werden, z. B. beim wöchentlichen Nahrungsmitteleinkauf. Bei nicht allein wohnenden Spieler/innen wird die eigene Spielpraxis vertuscht, z. B. durch nächtliches Spielen und/oder in der Kommunikation gegenüber den sozialen Kontrollinstanzen gänzlich ausgeklammert.

- «das kriegt keiner mit»/ «ich spreche gar nicht darüber».

Neutralisierungsstrategien. Greifen diese handlungspraktischen Strategien nicht (mehr) und wird das Konfliktpotenzial der abhängigen Spielpraxis für den oder die Spielende selbst oder sein/ihr soziales Umfeld sichtbar, so werden die hier als eigentliche Neutralisierungsstrategien ( $\mathrm{vgl}$. techniques of neutralization nach Sykes und Matza 1957) bezeichneten, weniger auf Handlungs- und stärker auf Deutungsebene ansetzenden Techniken bedeutsam. Sie werden bewusst oder unbewusst eingesetzt, um den Konflikt gerade nicht handlungsleitend im Sinne eines Karriereabbruchs werden zu lassen - im Sinne der Selbstvergewisserung: «warum Computerspielen (für mich) trotzdem gut ist». Diese Neutralisierungsstrategien werden in Abbildung 1 anhand zweier dimensionalisierender Achsen eingeordnet. Die horizontale Achse bezeichnet dabei, in welcher Weise sich die

2 Auf eine Schilderung der rekonstruktiven Methodik bei der Arbeit mit den Transkripten und Einzelfallrekonstruktionen wird hier verzichtet, da diese vielfach ausführlich (u.a. Bleckmann, Eckert, und Jukschat 2012; Bleckmann und Jukschat 2015; Jukschat 2014; Jukschat 2016) dargestellt wurde. 
Neutralisierungstechnik zur sozialen Kontrollinstanz bzw. den durch sie vertretenen sozialen Normen und Werten verhält, nämlich zwischen Anerkennung (links) und Nicht-Anerkennung (rechts). Die vertikale Achse steht für die Art und Weise, in der die Spieler ihre Erfahrung von Abweichung neutralisieren, oben die defensiven Formen, unten die offensiven Formen. Jede der Neutralisierungsstrategien wird mit einem kurzen Titel benannt (jeweils fett), innerhalb der Abbildung 1 eingeordnet, kurz beschrieben, ggf. mit Transkriptauszügen (jeweils kursiv) illustriert, jedenfalls aber stets zum Abschluss mit einer charakteristischen, verkürzten Aussage zur Wiedererkennung (jeweils mit bullet point) versehen.

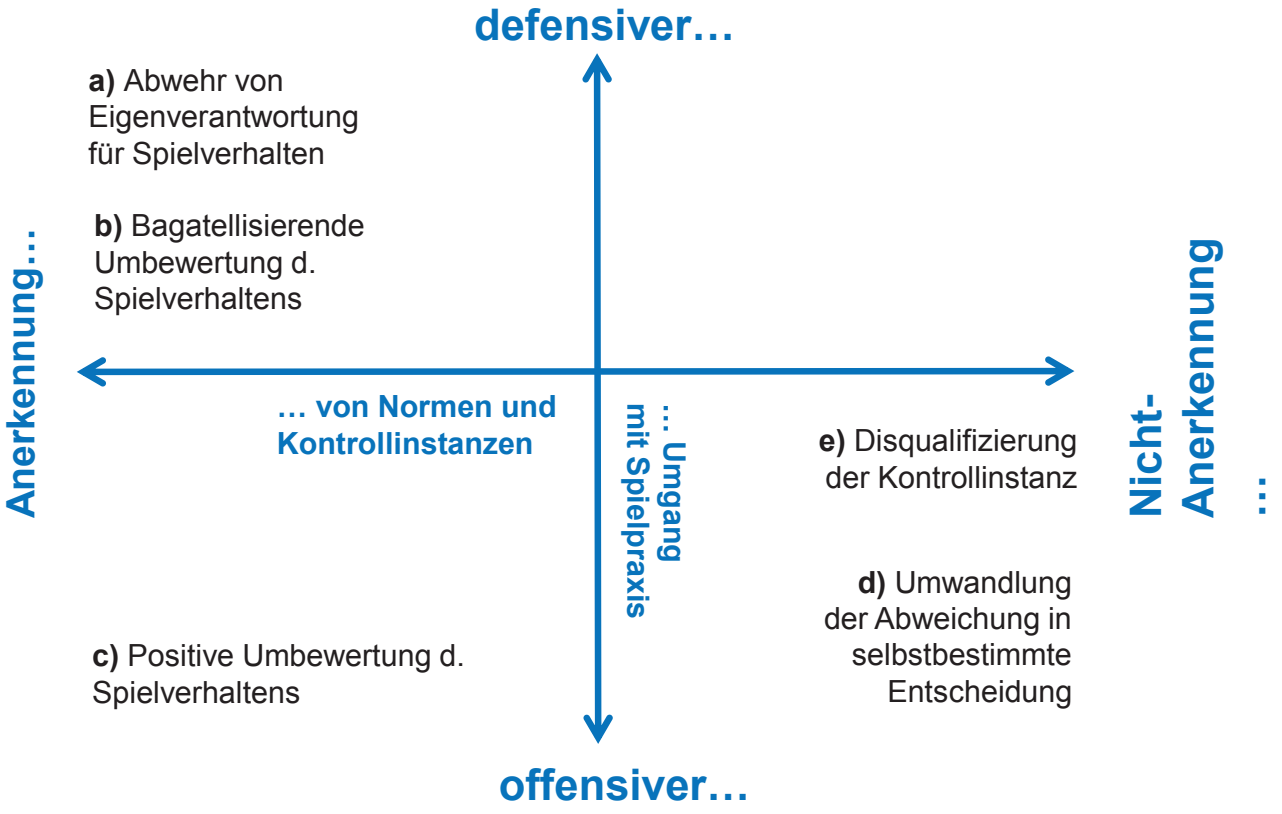

Abb. 1.: Neutralisierungsstrategien von suchtartig Computerspielenden im Überblick.

\section{a) Abwehr von Eigenverantwortung für das Spielverhalten}

Diese Neutralisierungsstrategie ist durch eine starke Anerkennung der gesellschaftlichen Werte und Normvorstellungen sowie ihrer Kontrollinstanzen charakterisiert und es wird ausgeprägt defensiv auf der Erfahrung von Abweichung reagiert. Spieler/innen, die diese Neutralisierungstechnik aufweisen, problematisieren ihre Spielpraxis zumeist selbst und bezeichnen sich als computerspielsüchtig. Sie weisen jedoch jegliche persönliche und individuelle Verantwortung zurück, indem sie beispielsweise auf externe Umstände oder süchtig machende Spielstrukturen als Ursache verwiesen oder biologistische Erklärungen für ihr Verhalten anführen. Die für diese Neutralisierungstechnik charakteristische Grundaussage lautet:

- «ich bin nicht schuld daran/lch kann nicht anders». 


\section{b) Bagatellisierende Umdeutung des Spielverhaltens.}

Das zweite in diesem Quadranten verortete Muster ist etwas weniger defensiv. Die Abweichung kann dabei erstens durch den Vergleich mit anderen, schlimmeren Fällen bagatellisiert werden, und/oder den Verweis, dass die derzeitige eigene Spielpraxis keine negativen Auswirkungen auf andere Lebensbereiche habe, die Funktionsfähigkeit also noch erhalten sei:

[...] hab ich vom Gymnasium auf die Sekundarschule gewechselt, dadurch konnte ich halt auch weiterhin ganz normal spielen, [...] sechs, sieben Stunden am Tag, ohne dass meine schulischen Leistungen gelitten haben. (Tim, 20)

Die Bagatellisierung erfolgt jedoch auch durch Abwehr einer Pathologisierung des Spielverhaltens, indem ein Vergleich mit anderen Tätigkeiten herangezogen wird, die von anderen Personen ausufernd ausgeübt würden, ohne dass dies als problematisch bewertet werde

[...] das ist vielleicht krankhaft, aber [...] andere sammeln Deckel von Milchkännchen, und fahren Wochenenden irgendwo hin, in die Schweiz, um Milchkännchendeckel auszutauschen. [...] Und das auch exzessiv. Da sagt keiner, dass die süchtig sind. (Ben, 28)

- «bei mir ist es nicht so schlimm (wie bei Person/Tätigkeit xy)»/ "das machen jetzt alle»/ «ich bin nicht süchtig»

\section{c) Positive Umdeutung des Spielverhaltens}

Dies ist die dritte Neutralisierungstechnik, die die sozialen Kontrollinstanzen und die durch sie vertretenen sozialen Normen und Werten ebenfalls grundsätzlich anerkennt. Der Umgang mit dem eigenen Spielverhalten ist jedoch sehr offensiv. Das Computerspielverhalten wird als eine Möglichkeit interpretiert, bestimmte gesellschaftlich geteilte Werte zu erreichen, oder sollten auch Wirkungen entstehen, die diesen Werten entgegenstehen, so werde jedenfalls in der Summe eine positive Wirkbilanz erzielt. Hierfür werden negative Effekte angezweifelt oder jedenfalls in ihrer Bedeutung heruntergespielt, und positive Effekte des Computerspielens auf den Erwerb von Fähigkeiten betont, die gesellschaftlich positiv bewertet werden, wie z. B. Sprachkenntnisse oder die Fähigkeit zu strategischem Denken.

- «ich lerne so viel anderes durch das Spiel»/ «lch lerne mich gut in den Medien zurechtzufinden». 


\section{d) Umwandlung der Abweichung in eine selbstbestimmte Entscheidung}

Standen bisher jene Neutralisierungstechniken im Fokus, die an bestimmte gesellschaftlich geteilte Vorstellungen und Erwartungen anschliessen und die sie vertretenden sozialen Kontrollinstanzen grundsätzlich anerkennen, kommt nun die erste Neutralisierungstechnik in den Blick, die das durch die Spielpraxis entstehende Konfliktpotenzial neutralisiert, indem sie diese nicht anerkennt. Die Spielenden deuten ihre Normabweichung in eine selbstbestimmte Entscheidung um, indem sie bestimmte gesellschaftlich geteilte Werte etwa mit Verweis auf ihre eigenen Bedürfnisse («ich brauch das nicht») in ihrem Geltungsbereich für sich zurückweisen. So wird angeführt, dass man sich ohnehin alleine ohne bindende Sozialbeziehungen wohler fühle oder ohnehin nicht nach beruflichem Erfolg strebe.

- «ich bin eben anders»/ «ich bin kein Workaholic»/«ich bin sowieso lieber für mich»

\section{e) Disqualifizierung der Kontrollinstanz}

Etwas weniger offensiv wird bei dieser Neutralisierungsstrategie mit der Abweichung umgegangen. Hier ist es eher die Kontrollinstanz selbst, die disqualifiziert wird. Damit wird die Aufmerksamkeit in der Auseinandersetzung weg von einer möglichen Problematik des eigenen Verhaltens gelenkt und/oder inhaltlich begründet, warum der Kontrollinstanz jegliches Recht abgesprochen werden muss, das eigene Spielverhalten zu beurteilen, es wird aber auch eine Disqualifizierung der Gesellschaft begründet, die diese Normen und Werte vertritt. Allerdings ist dabei oft eine Nähe zu den Strategien der positiven Umdeutung gegeben, wenn die Argumentationslinie lautet, dass nach Einschätzung des Spielenden die Vorteile gegenüber den Nachteilen der Spielpraxis klar überwögen, die Kontrollinstanz aber nicht in der Lage sei, diese Vorteile überhaupt zu erkennen und zu benennen:

Irgendwann wird das, was man in diesen Spielen leistet wirklich gesellschaftlich anerkannt. Dass man wirklich in eine Bewerbung schreibt, hier schaut, ich hab das und das im Spiel erreicht, hier ist der Ausdruck. Und dass die, die einen einstellen, dann halt schon wissen, so, hey (anerkennend), der kann auch mal 100 Stunden die Woche durchziehen. (Ben, 28)

- «meine Eltern haben mich sowieso auf dem Kieker»/ «Meine Lehrer haben keine Ahnung»/ "Die Gesellschaft versteht das nicht»

Insgesamt fällt über alle aufgezeigten Neutralisierungstechniken hinweg auf, dass sie das sich aus der suchtartigen Spielpraxis ergebende Konfliktpotenzial nur scheinbar auflösen. Bei den Analysen der Aussagen der Befragten treten bereits zum Zeitpunkt des möglichen Karriereabbruchs latent nach wie vor bestehende Widersprüchlichkeiten, Ambivalenzen sowie innere wie äussere Konflikte ans Licht (Jukschat 2014). 
Re-Evaluation der Neutralisierungsstrategien im weiteren Verlauf. Und wie geht es danach in der Karriere weiter? Das beschriebene Raster der Neutralisierungsstrategien wurde vorwiegend aus Interviews mit aktuell an diesem Punkt im Karriereverlauf befindlichen Personen rekonstruiert. Durch anschliessende Hinzunahme von Interviews mit ehemals suchtartig Spielenden konnte die Analyse erweitert werden. Eine Gemeinsamkeit ist die entweder vor der Reduktion des Spielverhaltens (Aussteiger) oder nachher (Ausschleicher) erfolgende kritische Re-evaluierung der eigenen neutralisierenden Argumentationslinien (vgl. Bleckmann und Fenner 2013). Re-Evaluation bedeutet in den meisten Fällen, dass die ausufernde Computerspielpraxis rückblickend erheblich negativer bewertet wird, kann aber zugleich zusätzlich durch ein positives Ergreifen von Lernpotentialen auf Meta-Ebene erfolgen, z. B. bei der Erstellung wissenschaftlicher Arbeiten zum Thema suchtartige Computerspielnutzung, die das Erlebte verarbeiten und einordnen helfen. Eine rückblickend vielleicht übertrieben negative Bewertung der eigenen Spielpraxis kann dabei auch im Sinne einer Rückfallprophylaxe gedeutet werden.

- «ich habe mir damals selbst etwas vorgemacht»/«ich verstehe warum das damals für mich Sinn gemacht hat»/«ich habe viel daraus gelernt».

\section{Parallelen zu Argumentationslinien von Medienkonzernen und -forschern?}

Als Sherry Turkle ihr Buch «Alone together» (2011) veröffentlichte, hätte man annehmen können, durch ihre Hinweise auf nachteilige Auswirkungen galoppierender technologischer Entwicklungen auf das menschliche Sozialverhalten kämen auch bisherige Technik-Euphoriker ins Grübeln, ins Bremsen, ins Abwägen. Technik-Euphoriker könnten die Professorin des Massachusetts Institute of Technology (MIT) und Techniksoziologin durchaus als seine von ihnens betrachten. Aus der Tatsache, dass in einer Forschungstradition, die sich seit Jahrzehnten mit der Rezeption neuester technologischer Entwicklungen befasst, nun (auch) problematische Auswirkungen gesehen werden und ein Umdenken empfohlen wird, könnte geschlossen werden, dass sich die Zeiten geändert haben, dass Risiken und Probleme tatsächlich zunehmen, Auswirkungen der technologischen Entwicklung tatsächlich immer mehr in Konflikt mit bestehenden Werte- und Normensystemen treten. Turkle, die es mit ihren früheren Werken bis auf die Titelseite von «Wired» geschafft hatte, berichtete nun in einem Interview, "Wired» habe ihr neuestes Buch nicht einmal rezensiert (de Lange 2013). Sie ist jetzt «[...] the one-time «cyber-diva» who some now call a «technophobe»» (ebd.), so der Titel des Artikels. Ist Sherry Turkle wirklich technophob geworden? Sie selbst attestiert sich keine Technophobie, sondern ein auf zahlreichen, auch eigenen Forschungsbefunden basiertes Umdenken. Aber: Wenn schon eine der weltweit führenden Techniksoziologinnen öffentlich als technophob bezeichnet wird, sobald sie (auch) einmal Einschränkungen der Nutzung 
empfiehlt, wäre für andere Forscher jedenfalls ein Grund zur Angst vorhanden, die innerhalb bestimmter Professionen eine «Deformation professionelle» begünstigen würde, "die dazu führt, dass aus der Angst, als Kulturpessimistln gelabelt zu werden, geradezu reflexhaft jegliche mediale Artikulation und Nutzungsform überbewertet und bei öffentlicher Kritik verteidigt wird» (Kommer 2014, 2), wie es für die Medienpädagogik als Befürchtung bereits formuliert wurde. Das wäre eine besorgniserregende Entwicklung. Ebenso besorgniserregend wäre andererseits ein reflexartiges Zustimmen zu einer möglicherweise unbegründeten Medienschelte. Die Idee, im Diskurs um Chancen und Risiken von Computerspielen Parallelen zu den Neutralisierungsstrategien suchtartiger Computerspieler herauszupicken, könnte hilfreich sein, um den Blick für das möglicherweise reflexhaft positiv Überbewertende in eigenen Argumentationslinien zu schärfen. Um bei dieser Schärfung die Abgrenzung zu Positionen des oftmals auch von den Kommerzialisierungsinteressen grosser Medienanbieter dominierten öffentlichen Diskurses zu ermöglichen, werden nicht nur Parallelen zur Forschungslandschaft, sondern auch aus der öffentlichen Diskussion in den Medien herangezogen. Vor dem Einstieg in die Beschreibung ausgewählter Parallelen sollen zwei wichtige Einschränkungen dieser Vorgehensweise genannt werden: Erstens, es wird nicht der Anspruch erhoben werden, dass die Parallelisierung sich auf eine systematische und umfassende Darstellung einer Position «der Medienpädagogik» beziehen würden, allein schon weil deren grosse Traditionslinien (grob: Mediensozialisationsforschung, Medienkompetenzforschung, Mediendidaktik) mit ihren sehr unterschiedlichen zugrundeliegenden Theoriebezügen bereits nur sehr bedingt miteinander vereinbare Positionen im Diskurs einnehmen. Die Medienkompetenzforschung und die Mediendidaktik zeigen eher Parallelen zu den Neutralisierungsstrategien der Bagatellisierung und positiven Umdeutung auf der linken Seite, die Mediensozialisationsforschung eher zu Neutralisierungsstrategien auf der rechten Seite, unter Ablehnung des Normen- und Wertesystems. Ziel ist also nicht eine umfassende Parallelisierung von Handlungs- und Argumentationsmustern, sondern die Anregung zum (selbstkritischen) Weiterdenken. Zweitens, um es noch einmal zu wiederholen, ist den oben beschriebenen Neutralisierungsstrategien gerade nicht gemeinsam, dass sie an sich falsch oder selbsttäuschend wären. Gemeinsam ist ihnen auf dem Weg einer immer stärker das Leben dominierenden Spielpraxis die Funktion der Verhinderung eines Karriereabbruchs. Doch nun zur Parallelisierung:

Isolation/Vertuschung. Kann eine ausschliessliche Fokussierung auf In-Game-Verhaltensweisen in der wissenschaftlichen Untersuchung von Computerspielverhalten als Parallele zur Konfliktvermeidung durch Isolation bzw. Vertuschung angesehen werden? In der Forschungstradition der Game Studies lag lange Zeit und z. T. auch heute noch der einzige Fokus auf dem In-Game-Verhalten von Computerspielenden. Insofern konnten Konflikte, die sich zwischen der Spielpraxis und der 
Ausbildung, dem Beruf, den Beziehungen, anderen Hobbies oder der Gesundheit der Computerspieler ergeben haben, überhaupt nur insoweit als Untersuchungsgegenstand der Game Studies bearbeitet werden, wie sie von den Spielern selbst 〈ins Spiel hineingeholts und In-Game thematisiert wurden.

Abwehr von Eigenverantwortung. Inzwischen wird auch von Computerspielherstellern die Existenz suchtartiger Nutzungsformen meist bejaht, nachdem über Jahrzehnte die bagatellisierende Umdeutung (s.u.) die dominante Argumentationslinie war. Zeichnet sich hier wiederum eine Parallele zu den beschriebenen Neutralisierungsstrategien der Computerspieler selbst ab? Die Verantwortung für die suchtartige Nutzung wird von den Spieleherstellern jedenfalls nicht auf der Seite der Spiele und ihrer Spielstruktur gesehen, sondern in einer kranken Persönlichkeitsstruktur oder im ungünstigen Umfeld. Bei jugendlichen Spielern werden dabei in Publikationen der Computerspielverbände explizit die Eltern als Verursacher genannt: "eine Suchtgefährdung entsteht vor allem dann, wenn der Erziehungsauftrag in verschiedenen Bereichen nicht verantwortungsbewusst wahrgenommen wird», so der GAME in seiner Broschüre «Richtig Spielen».

Findet sich etwa noch eine ganz andere Variante der Abwehr von Eigenverantwortung, und zwar in der Medienpädagogik, wenn am Beginn vieler Veröffentlichungen steht, die Computerspiele bzw. allgemeiner digitale Medien gehören zum Alltag von Kindern heute einfach dazu (exemplarisch etwa das JFF - Institut für Medienpädagogik in Forschung und Praxis o. J. auf seiner Homepage)? Man verweist auf die aktuellen Nutzungsdaten aus den KIM-Studien. Das durchschnittliche Ausmass der familiären Bildschirmmediennutzung, besonders in benachteiligten sozialen Schichten, wird dabei in der Regel nicht gutgeheissen, sondern kritisiert, jedoch als durch Medienpädagogik nicht beeinflussbar angesehen, nach dem Motto «es gibt ein Problem, aber wir können daran nichts ändern»/ «alle machen das jetzt».

Bagatellisierende Umdeutung. Weisen vielleicht auch die historisch älteren Argumentationslinien der Medienhersteller, in denen ausuferndes Computerspielverhalten verharmlost wird, Parallelen zu den Neutralisierungsstrategien der suchtartigen Computerspieler selbst auf? Jedenfalls finden sich in der öffentlichen Debatte in den Medien wie auch in den Darstellungen der Computerspielhersteller und ihrer Verbände ähnlich wie der oben erwähnte «Milchkännchendeckel-Sammel-Vergleich〉 gehäuft Vergleiche zu anderen 〈Hobbies〉, die ebenfalls exzessiv ausgeübt, jedoch von der Gesellschaft nicht als problematisch bewertet würden, z.B. immer wieder der Vergleich mit Fussballspielen: «Warum sagt eigentlich nie jemand, dass ein Junge süchtig sei, wenn er jeden Nachmittag nach der Schule auf dem Fußballplatz verbringt? Weil er mit anderen zusammenspielt? Das tun Computerspieler auch» (Buse et al. 2014, 64). In diesen Vergleichen werden nicht ernsthaft zwei exzessiv ausgeübte Tätigkeiten genau charakterisiert und bzgl. ihrer 
langfristigen Auswirkungen verglichen, sondern der Vergleich wird eher als rhetorische Figur angeführt.

Positive Umdeutung. Könnten Argumentationslinien in der Öffentlichkeit, die die Chancen des Comuterspielens hervorheben und die Risiken ausblenden oder abschwächen, als Parallelen zur Neutralisierungsstrategie der positiven Umdeutung gezählt werden? In den Publikationen der Computerspielhersteller und ihrer Verbände finden sich zahlreiche Darstellungen mit Betonung von Chancen und $\mathrm{Ne}$ gierung oder Abschwächung der Risiken der Computerspielnutzung, z. B. in der USK-Plakatkampagne "Games und Gamer - ich spiele auch», die inzwischen nicht mehr online, aber in dem von dem Medienpädagogen Thomas Feibel herausgebenden Elternratgeber zum Thema «Kind und Computerspiele» (2005) abgebildet ist. Eine der extremsten Betonungen positiver Computerspieleffekte findet sich in dem Buch «Besser als die Wirklichkeit» der Gamedesignerin McGonigal (2012). Computerspiele machten schlau, gesund, sportlich, schön, hilfsbereit, innovativ, glücklich und sozialkompetent, um nur einige der von ihr vertretenen mehr als ein Dutzend Behauptungen zu nennen, die in Form kurzer, als «Fixes» bezeichneter Thesen formuliert werden.

Negative Medienwirkungen werden ausgeblendet. Ebenfalls als Parallele zur Neutralisierungsstrategie der positiven Umdeutung könnte die Positionierung medienpädagogischer Institutionen zu 〈medieneuphorischen〉 wie «medienkritischen> Publikationen bezeichnet werden. Dies sei hier einmal exemplarisch an den Reaktionen des Landesmedienzentrums in Baden-Württemberg auf zwei Bücher dargestellt, McGonigals o. g. «Besser als die Wirklichkeit» und Spitzers «Digitale Demenz» (2012), in dem negative Auswirkungen der Nutzung von Bildschirmmedien auf Schulleistungen, Schlaf, Übergewicht etc. dargestellt werden. In der Stellungnahme zu Spitzer finden sich die folgenden Wörter und Phrasen: «verkürzt, falsch, demagogisch, billig, weltfremd, monokausal, Verdrehung der Tatsachen, vielfach fehlen Belege» (Bounin 2012). McGonigals Buch dagegen wird vom LMZ nicht kommentiert, sondern in der Rubrik «Wie wirken Computerspiele?» im Sinne einer kurzen Inhaltsübersicht dargestellt (Heinz O.J.).

Zwei methodische Randbemerkungen seien an dieser Stelle erlaubt:

Erstens: Es scheint hier mit zweierlei Mass gemessen zu werden: ein hoher wissenschaftlicher Anspruch wird eingefordert, wenn negative Wirkungen behauptet werden, die auf diese Weise entkräftet werden können. Wieso kann dieser Anspruch bei den positiven Wirkungen entfallen?

Und zweitens: In der Mediendidaktik wird immer wieder gefragt, inwieweit eine Übertragung, des am Bildschirm Gelernten, langfristig ins Leben gelingt (beim Gaming: intermondialer Kompetenztransfer). Einen solchen Transfer nehmen die Computerspielenden implizit an, wenn sie über ihre Lernerfolge, z. B. in Englisch, berichten. In einer Studie von Lampert und Kollegen werden Items abgefragt wie: 
"Weil ich solche Spiele spiele, fällt es mir leichter, auf andere Menschen zuzugehen.» "Seit ich solche Spiele spiele, kann ich mich auch ausserhalb des Spiels besser auf bestimmte Dinge konzentrieren.» Bei Vielspielern ist dabei die Zustimmung zu Selbsteinschätzung eines gelingenden Transfers deutlich höher als bei Gelegenheitsspielern (Lampert et al. 2011). Aber Achtung: Dies erscheint konsistent mit der Deutung der Zustimmung als positiv umdeutende Neutralisierungsstrategie, ist aber keinesfalls ein stichhaltiger Beleg für einen tatsächlich stattfindenden Kompetenztransfer.

Umdeutung in selbstbestimmte Entscheidung. In der Mediensozialisationsforschung wird das Medienhandeln von Kindern und Jugendliches über längere Zeiträume aus der Sicht der Handelnden ausführlich charakterisiert, und daraufhin untersucht, welche Rolle es bei Prozessen der Emanzipation, der Identitätskonstruktion, der Herausbildung subversiver Jugendkulturen einnimmt. Die stark qualitativ-biographisch orientierte Forschungsmethodik dieser Disziplinen führt mitunter zu einer Pauschalabwehr aller quantitativen Methoden der Medienwirkungsforschung inklusive der gesamten medizinisch-psychologischen Computerspielsuchtforschung. Diese beruhten auf der falschen Frage («was machen die Medien mit dem Menschen?»), man wisse aber inzwischen um die Bedeutung individuellen Medienhandelns, die richtige Frage laute also «Was machen die Menschen mit den Medien?»). Hier könnte eine Parallele zu den Neutralisierungsstrategien gesehen werden, bei denen die Computerspieler deutlich machen, dass sie nicht aus Zwang, sondern aufgrund ihrer eigenen selbstbestimmten Entscheidung, sich nicht an den Normen des sozialen Umfelds bzw. der Gesellschaft zu orientieren, immer mehr Computerspiele spielen.

Disqualifizierung der externen Kontrollinstanzen. Ähnlich wie die Eltern oder Partner der Computerspieler als Kontrollinstanzen disqualifiziert werden («die haben sowieso keine Ahnung»), fällt in der öffentlichen Debatte als mögliche Parallele die Disqualifizierung der Person der Autoren (epikur 2009) als Reaktion auf Veröffentlichungen von diesen Autoren auf, in denen auf Gefahren und Probleme der Computerspielnutzung hingewiesen wird. Ebenso könnte man die LabellingDebatte, s. o. (Wimmer 2013, 93), an dieser Stelle einordnen. Ein Problem hätten dabei nicht die intensiv Computerspielenden, sondern die Gesellschaft, die deren Verhalten nicht versteht.

\section{Fazit}

Die Impulse zur kritischen Selbstreflexion der Medienpädagogik, die sich durch die Beispiele der ausgewählten Parallelisierungen ergeben könnten, gehen u. E. in zwei gänzlich unterschiedliche Richtungen: Der Blick auf die linke Seite in der Abb. 1 unterstreicht, wie wichtig es ist, den vielleicht noch aus historischen Traditionen 
abgeleiteten Wunsch bzw. die Annahme der Medienpädagogik, durch Computerspiele doch zumindest in der Bilanz überwiegend positive Effekte zu erzielen, durch eine saubere wissenschaftliche Methodik (umfassende Literaturrecherche, Beleg durch peer-review Veröffentlichungen, alternative-treatment Kontrollgruppen, Längsschnittdaten, Berücksichtigung vieler Variablen in einem komplexen Wirkmodell, Offenlegung von Interessenkonflikten, Untersuchungen auch an besonders vulnerablen Gruppen, Nachweis des intermondialen Kompetenztransfers etc.) abzusichern, und dabei nicht, wie oben beschrieben, je nach Erwünschtheit des Outcomes mit zweierlei Mass zu messen. Einen Schritt in die richtige Richtung stellt dabei u.E. z.B. das zunehmende «Hereinholen der realweltlichen Biographie» und die Bearbeitung der dadurch sichtbar werdenden Konflikte in die Game Studies dar. Auf der rechten Seite dagegen ist vielleicht eher die typische Gefahr einer Subkulturforschung gegeben: Wenn die untersuchte Subkultur wächst, gedeiht, finanzkräftig wird, kann sie Teile der sie untersuchenden Forschungsgemeinschaft für ihre Zwecke instrumentalisieren, die ihr mit ihrer wissenschaftlichen Credibility helfen, den Anstrich des Subversiven zu behalten. Wo immer Konflikte zwischen einer Computerspielpraxis und der Gesundheit, der Bildungs- oder Berufsbiographie, der Beziehung etc. aufscheinen, können sie problemlos mit Verweis darauf, das seien Werte, die die Subkultur ohnehin nicht teile, neutralisiert werden. Die milliardenschwere Games-Industrie ist zwar längst Mainstream, aber den alten Anstrich zu behalten, könnte sich für sie zusätzlich noch lohnen, allein schon weil für das Marketing an jugendliche Zielgruppen gerade dieses Subversive, die Atmosphäre der Abgrenzung von gesellschaftlichen Normen einen Vorteil darstellen dürfte. Die Medienpädagogik muss sich an dieser Stelle tatsächlich fragen, ob die «Forschung und Theoriebildung nicht streckenweise zu einer mehr oder weniger unreflektierten Affirmation des je aktuellen medienkulturellen oder medientechnischen Hypes» (Kommer 2014, 2) regrediert ist.

\section{Literatur}

American Psychiatric Association. 2013. «Diagnostic and Statistical Manual of Mental Disorders Fifth Edition DSM-5TM».

Becker, Howard S. 1963. Outsiders. Studies in Sociology of Deviance. New York: The Free Press.

Bleckmann, Paula, Judith Eckert, und Nadine Jukschat. 2012. «Futile search for a better life? Two biographical case studies on women with depression and video game dependency». Advances in Dual Diagnosis 5 (3): 137-146.

Bleckmann, Paula, und Irmela Fenner. 2013. "Verankerung und Vertreibung in realen und virtuellen Welten. Biographische Längsschnittinterviews zu Bewältigung bei Computerspielsucht». BIOS - Zeitschrift für Biographieforschung, Oral History und Lebensverlaufsanalysen 26 (1): 77-109. 
Bleckmann, Paula, und Nadine Jukschat. 2015. «The Integrated Model of (Dys-) Functionality: Reconstructing Patterns of Gaming as Self-Medication in Biographical Interviews with Video Game Addicts». FQS 16 (3).

Bounin, Ingrid. 2012. «Stellungnahme zu Manfred Spitzers Thesen». https://www.Imz-bw.de/ medienbildung/aktuelles/mediaculture-blog/blogeinzelansicht/2012/stellungnahmezu-manfred-spitzers-thesen.html.

Buse, Uwe, Friederike Schröter, und Jonathan Stock. 2014. «Du sollst spielen!» Der Spiegel, Nr. 3: 60-67.

Chappell, Darren, Virginia Eatough, Mark N. O. Davies, und Mark Griffiths. 2006. «EverQuest_-It's Just a Computer Game Right? An Interpretative Phenomenological Analysis of Online Gaming Addiction». International Journal of Mental Health and Addiction 4 (3): 205-216. doi:10.1007/s11469-006-9028-6.

Corneliussen, Hilde G., und Jill Walker Rettberg. 2008. Digital Culture, Play, and Identity. A World of Warcraft Reader. Cambridge, Mass.: The MIT Press.

Cover, Rob. 2006. "Gaming (Ad)diction: Discourse, Identity, Time and Play in the Production of the Gamer Addiction Myth». Games studies the international journal of computer game research 6 (1). http://gamestudies.org/0601/articles/cover.

Dellwing, Michael. 2013. «Addiction Diagnoses as Involvement Controls: The Pragmatic Value of Psychiatric Definitions of the Situation». RESET - Recherches en sciences sociales sur Internet 1 (2): 103-129.

Dollinger, Bernd. 2002. Drogen im sozialen Kontext. Zur gegenwärtigen Konstruktion abweichenden Verhaltens. Augsburg: MaroVerlag.

Dollinger, Bernd, und Henning Schmidt-Semisch, Hrsg. 2007. Sozialwissenschaftliche Suchtforschung. Wiesbaden: VS Verlag für Sozialwissenschaften.

Domahidi, Emese, und Thorsten Quandt. 2014. «〈And all of a sudden my life was gone....: A biographical analysis of highly engaged adult gamers». New Media \& Society, Februar. doi:10.1177/1461444814521791.

epikur. 2009. «Pfeiffer die Pfeife». http://www.zeitgeistlos.de/zgblog/2009/pfeiffer-die-pfeife/.

Gerhardt, Uta. 1991. «Krankheits- und Patientenkarrieren». In Handbuch Qualitative Sozialforschung. Grundlagen, Konzepte, Methoden und Anwendungen, hrsg. v. Uwe Flick, Ernst v. Kardorff, Heiner Keupp, Lutz v. Rosenstiel, und Stephan Wolff, 312-316. München: Psychologie Verlags Union.

Gerstenberger, K.-Peter, Marek Klingelstein, und Thomas Feibel, Hrsg. 2005. Crashkurs Kind und Computerspiele. 1., Aufl. Stuttgart: Klett, Ernst, Verlag GmbH.

Goffman, Erving. 1977. «Die moralisch Karriere des Geisteskranken». In Asyle. Über die soziale Situation psychiatrischer Patienten und anderer Insassen, hrsg. v. Erving Goffman, 3:125-167. Frankfurt am Main: Suhrkamp Verlag.

Heinz, Daniel. o. J. «Wie wirken Computerspiele?» https://www.Imz-bw.de/wirkung-computerspiele.html.

Hussain, Zaheer, und Mark D. Griffiths. 2009. "The Attitudes, Feelings, and Experiences of Online Gamers: A Qualitative Analysis». CyberPsychology \& Behavior 12 (6): 747-753. doi:10.1089/cpb.2009.0059.

JFF - Institut für Medienpädagogik in Forschung und Praxis. ○. J. «Medien gehören zum Alltag». zappen klicken surfen. http://www.zappen-klicken-surfen.de/?BE_ID=1. 
Jukschat, Nadine. 2013. «Extending the Study of Videogame Dependency: Social Meaningfulness of «Addictive〉 Gaming and Self-Analyses of Dependency.» RESET - Recherches en sciences sociales sur Internet 1 (2): 73-102.

Jukschat, Nadine. 2014. «Addictive Gaming: Self-analyses of addiction and techniques of neutralizing deviance». Vortrag gehalten auf der XVIII ISA World Congress of Sociology vom 13. bis 19. Juli, Yokohama, Juli 16.

Jukschat, Nadine. 2016. "Computerspielabhängigkeit als unwahrscheinliche Karriere. Weichenstellungen und Mechanismen im Prozess der Entstehung und Verstetigung. Erste Ergebnisse biografischer Fallanalysen». In Qualitative Gesundheitsforschung. Beispiele aus der Forschungspraxis, hrsg. v. Carsten Detka. ZBBS-Buchreihe. Studien zur qualitativen Bildungs-Beratungs-und Sozialforschung. 279-297. Verlag Barbara Budrich.

Kammerl, Rudolf, Lena Hirschhäuser, Moritz Rosenkranz, Christiane Schwinge, Sandra Hein, Lutz Wartberg, und Kay Uwe Petersen. 2012. EXIF - Exzessive Internetnutzung in Familien. Berlin: Bundesministerium für Familie, Senioren, Frauen und Jugend.

Kommer, Sven. 2014. "Call for Papers zur Frühjahrstagung. Spannungsfelder und blinde Flecken. Medienpädagogik zwischen Emanzipationsanspruch und Diskursvermeidung.»

Lampert, Claudia, Christiane Schwinge, und Sheela Teredesai. 2011. «Kompetenzförderung in und durch Computerspiele(n)». In Kompetenzen und exzessive Nutzung bei Computerspielern: gefordert, gefördert, gefährdet, hrsg. v. Jürgen Fritz, Jan-Hinrik Schmidt, und Claudia Lampert, 117-179. Schriftenreihe Medienforschung der LfM 66. Berlin: Vistas.

Lange, Catherine de. 2013. "Sherry Turkle: «We're losing the raw, human part of being with each others. Face to face with the one-time ucyber-divas who some now call a stechnophobes». http://www.theguardian.com/science/2013/may/05/rational-heroes-sherry-turkle-mit.

McGonigal, Jane. 2012. Besser als die Wirklichkeit!: Warum wir von Computerspielen profitieren und wie sie die Welt verändern. München: Heyne Verlag.

O'Brien, Scott. 2014. «Autism-related protein shown to play vital role in addiction». http:// www.eurekalert.org/pub_releases/2014-05/mh-aps050914.php.

Schmidt, Jan-Hinrik, Marius Drosselmeier, Wiebke Rohde, und Jürgen Fritz. 2011. «Problematische Nutzung und Abhängigkeit von Computerspielen». In Kompetenzen und exzessive Nutzung bei Computerspielern: gefordert, gefördert, gefährdet, hrsg. v. Jürgen Fritz, Jan-Hinrik Schmidt, und Claudia Lampert, 201-251. Schriftenreihe Medienforschung der LfM 66. Berlin: Vistas.

Spitzer, Manfred. 2012. Digitale Demenz. Wie wir uns und unsere Kinder um den Verstand bringen. München: Droemer Verlag.

Sykes, Gresham M., und David Matza. 1957. «Techniques of Neutralization: A Theory of Delinquency». American Sociological Review 22 (6): 664-670. doi:10.2307/2089195.

Turkle, Sherry. 2011. Alone together: Why we expect more from technology and less from ourselves. New York: Basic Books.

Walter, Susanne, und Michael Schetsche. 2003. «Internetsucht - eine konstruktionistische Fallstudie». Soziale Probleme. Zeitschrift für soziale Probleme und soziale Kontrolle 14 (1): 5-40.

Wimmer, Jeffrey. 2013. Massenphänomen Computerspiele. Konstanz und München: UVK Verlagsanstalt. 